\title{
Hemoptisis de repetición por aspiración bronquial de un cuerpo extraño: presentación de un caso y revisión de la literatura
}

\author{
M.C. ABELLÁN MARTÍNEZ, P. MÉNDEZ MARTÍNEZ, F. SÁNCHEZ GASCÓN, \\ J. HERNÁNDEZ MARTIINEZ, F.J. RUIZ LOPEZ \\ Servicio de Medicina Interna. Sección de Neumología. Hospital General Universitario. \\ Murcia
}

\begin{abstract}
RESUMEN
La aspiración bronquial de cuerpos extraños es más frecuente en niños. La etiología más usual son los cuerpos extraños de origen vegetal, que no se aprecian en una Rx simple de tórax. Las manifestaciones clínicas son variables, incluyendo la sensación de ahogo, tos y sibilancias. La hemoptisis es un síntoma infrecuente, relacionándose su aparición, generalmente, con la existencia de bronquiectasias.

Presentamos el caso de un niño que presentó hemoptisis de repetición y neumonitis local por Eikenella corrodens tres años después de la aspiración de una espiga. Se llegó al diagnóstico de la broncoaspiración tras la expulsión del cuerpo extraño, días después de la realización de un lavado broncoalveolar.

Lo inusual de este caso es la presentación clínica de hemoptisis de repetición, el largo periodo asintomático tratándose de un cuerpo extraño de origen vegetal y la neumonitis por Eikenella Corrodens asociada a la existencia de un cuerpo extraño intrabronquial.
\end{abstract}

PALABRAS CLAVE: Hemoptisis. Aspiración cuerpo extraño. Eikenella corrodens.
REPEATED HEMAOPTYSIS FOR FOREIGN BODY BRONCHIAL ASPIRATION: PRESENTATION OF ONE CASE AND REVIEW OF LITERATURE

\begin{abstract}
The foreign body aspiration is more frequent in children. The most usual etiology is the foreign bodies of vegetable nature, which can not be observed in the chest radiograph. The clinic signs are varied, inclu ding shortness of breath, cough and wheezing. Hemoptysis is a rare symptom, whose araising is generaly connected with the existence of bronchiectasis. We present the case of a child who showed repeated hemoptysis and focal neumonitis due to Eikenelle corrodens three years after the aspiration of a spike. The diagnosis of the bronchial aspiration obtained after The most unusual in this case is the clinic manifestation of repeated hemoptysis, together with the long connected to the existence of foreign body inside the bronchus.
\end{abstract}

KEY WORDS: Hemoptysis. Foreign body aspiration. Eikenella corrodens.

Abellán Martínez MC, Méndez Martínez P, Sánchez Gascón F, Hernández Martínez J, Ruiz López F.J. Hemoptisis de repetición por aspira ción bronquial de un cuerpo extraño: presentación de un caso y revisión de la literatura. An Med Interna (Madrid) 2000; 17: 652-654.

\section{INTRODUCCIÓN}

La aspiración bronquial de cuerpos extraños es más frecuente en niños, sobre todo en menores de 3 años (1).

Su diagnóstico puede complicarse si se desconoce el antecedente de la aspiración, lo que ocurre si el episodio sucedió hace mucho tiempo, si el paciente es un niño pequeño que estuviera sólo en ese momento o si el paciente presentara pérdida de conciencia o alteración de sus funciones mentales superiores. A esta dificultad hay que añadir la variedad en la presentación clínica.

Presentamos a continuación un caso clínico en el cual el diagnóstico de aspiración de un cuerpo extraño se obtuvo 3 años después tras estudio de episodios repetitivos de hemoptisis y neumonitis local por Eikenella Corrodens.

\section{CASO APORTADO}

Varón de 11 años de edad que consultó por hemoptisis. Entre los antecedentes personales destacaba la emisión de sangre por la boca 3 años antes de origen no determinado, no realizándose estudio radiológico. No tenía antecedentes de infecciones respiratorias de repetición, traumatismo torácico, patología renal ni anemia. No refería aspiración de cuerpos extraños. Tres semanas previas había presentado fiebre que cedió con tratamiento antibiótico, persistiendo la tos, acompañada en la última semana de esputos hemoptoicos. Consultó

Trabajo aceptado: 6 de Abril de 1999 
en el Servicio de Urgencias de este centro por hemoptisis de $150 \mathrm{cc}$, sin compromiso hemodinámico, y sin otra sintomatología acompañante.

En la exploración presentaba TA: 110/70 mmHg, FC: 90 lpm, Ta: $36^{\circ} \mathrm{C}$, FR: $12 \mathrm{rpm}$, con buena coloración de la piel y mucosas. La orofaringe era normal. No tenía IY, bocio, ni adenopatías. Los pulsos periféricos eran simétricos. La auscultación cardiaca era normal. En la auscultación pulmonar presentaba hipofonesis basal izquierda. El abdomen, la exploración neurológica y extremidades eran normales.

El hemograma, bioquímica, analítica de orina, coagulación, factor reumatoide, complemento, inmunoglobulinas, proteinograma, ANA y anticuerpo antimembrana basal fueron normales. Se realizó Mantoux que fue negativo. El EGC no presentó hallazgos patológicos. En la microbiología de esputo se aisló flora habitual.

Se realizó una Rx tórax apreciándose un infiltrado intersticio alveolar en lóbulo inferior izquierdo (Fig. 1). En la TAC torácica se apreciaba condensación en segmento basal posterior y lateral del LII, con broncograma aéreo (Fig. 2). En el estudio funcional respiratorio destacaba únicamente una disminución moderada de la difusión (D/VA: 65\%).

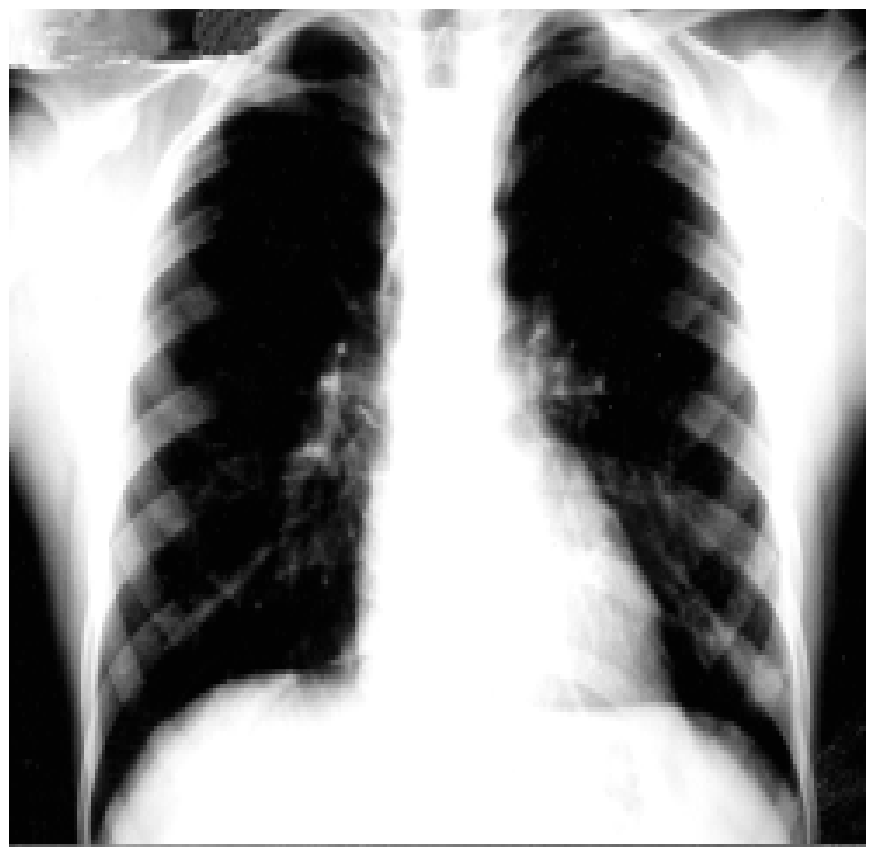

Fig. 1. Rx de tórax PA. Condensación en lóbulo inferior izquierdo.

Se realizó una broncoscopia objetivándose una mucosa con signos inflamatorios en bronquio principal izquierdo, y sobre todo, en la pirámide basal, con secreción mucosa blanquecina a nivel de $S_{9}$.

En el examen microbiológico del aspirado bronquial se aisló Eikenella Corrodens, y el estudio citológico mostró hemosiderófagos.

Ante los hallazgos broncoscópicos, a la terapia de control de la hemoptisis se asoció tratamiento antibiótico (Amoxicilina-Clavulánico) y se repitió la TAC a las tres semanas, observándose que, aunque había mejorado el infiltrado, persistía la imagen radiológica.

El paciente, que había continuado con una tos seca, de predominio nocturno, ingresó de nuevo un mes después por hemoptisis de 250 cc. sin repercusión hemodinámica. Se realizó ecocardiograma, no objetivándose alteraciones. Se repitió la broncoscopia observándose las mismas alteraciones vistas anteriormente. Se realizó un lavado broncoalveolar en $\mathrm{S}_{9}$.

El paciente, tras varios días asintomático, se remitió a su domicilio en espera de resultados pendientes. A los 2-3 días de su alta, pre-

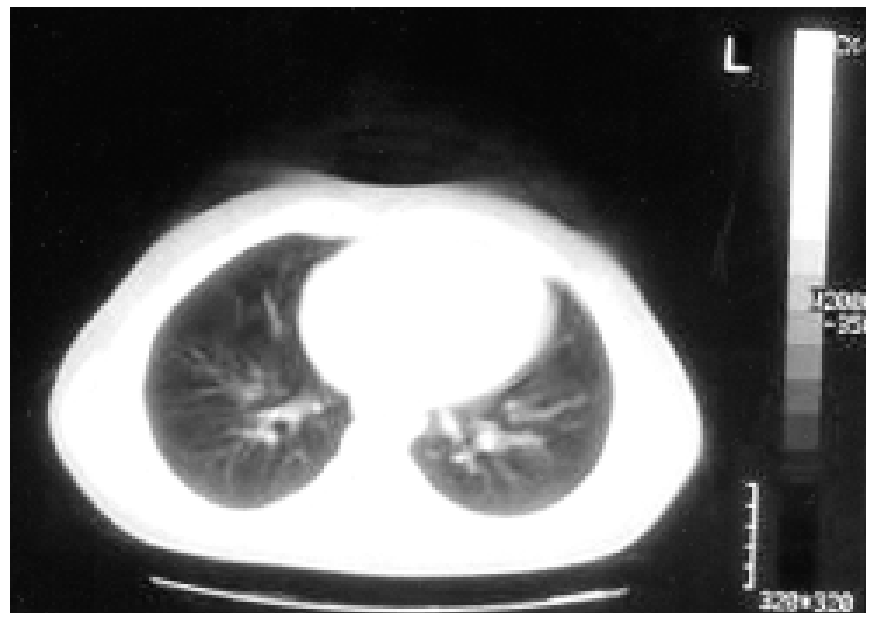

Fig. 2. TAC torácica. Infiltrado alveolar con broncograma aereo a nivel del lobulo inferior izquierdo, segmento 9.

sentó tos intensa eliminando un cuerpo extraño de origen orgánico, en concreto, una espiga.

Al insistir a los familiares sobre el antecedente de la inhalación, recordaron que 3 años antes aspiró hierbas mientras jugaba en el campo.

\section{DISCUSION}

Los cuerpos extraños que provocan obstrucción bronquial son los de pequeño tamaño, que atraviesan la laringe. El origen de los cuerpos extraños descritos en la literatura incluye dientes, huesos (2), lápices, alfileres, agujas, termómetros, cristal (3), joyas, caramelos, fármacos (4), caparazón de gambas (5) y sustancias vegetales, siendo éstas últimas las más frecuentes (1). Dentro de estas sustancias, las que se encuentran con mayor frecuencia son los cacahuetes. En el caso que presentamos, el cuerpo aspirado era una espiga.

Aparecen ya descritos en la literatura casos de inhalación de cabezuelas en flor de hierbas $(3,6)$. Las que presentan espigas suelen progresar más periféricamente, llegando incluso al espacio pleural (7) y dando lugar a una fístula broncopleurocutánea. Cuando el material inhalado es un vegetal puede absorber líquido e hincharse, obstruyendo en mayor o menor grado la vía aérea.

Las características patológicas durante la fase aguda comprenden edema e infiltrado inflamatorio. Esta respuesta tiende a producirse a las pocas horas, o como mucho a los pocos días, con obstrucción de las vías aéreas. Si se produce necrosis aparece tejido de granulación en la zona de contacto con el cuerpo extraño. Si la retención es crónica, se provoca fibrosis y estenosis de la pared bronquial, ocasionando bronquiectasias distales y neumonitis obstructiva.

La clínica después de la aspiración de los cuerpos extraños intrabronquiales consiste en tos, sibilancias, hipoventilación y roncus, que suele producirse a las pocas horas tras la aspiración. Sin embargo, puede existir un intervalo asintomático de meses o años si los bronquios no están totalmente obstruidos y sobre todo, si el material es hueso o materia inorgánica (8).

Los pacientes suelen recordar el episodio de ahogo relacionado con la aspiración, pero en ocasiones es necesario insistir en este hecho, sobre todo si ocurrió hace mucho tiem- 
po. En niños que presenten tos y sibilancias durante el verano, hay que descartar la aspiración de hierbas y hacer un diagnostico diferencial con el asma (9).

La aspiración de cuerpos extraños es una causa infrecuente de hemoptisis masiva. En un estudio de 264 broncoscopias por hemoptisis, ninguna fue por cuerpo extraño (10). En otro estudio de 60 pacientes se encontró 9 casos en que estaba provocada por cuerpo extraño (11). En el caso de aspiración de cuerpos extraños, la hemoptisis se asocia generalmente a la existencia de bronquiectasias.

La Rx tórax debe realizarse en espiración completa para demostrar el atrapamiento aéreo. En niños muy pequeños es útil hacer un decúbito lateral sobre el lado afecto, con lo que la movilidad del hemitorax es menor, apareciendo hiperclaridad y aumento de volumen en esa zona afectada (12). Generalmente se afectan los lóbulos inferiores. El hemitórax más afectado en adultos es lado derecho (1). Sin embargo, en los niños según Cleveland, es en el lado izquierdo y lo atribuye a la igualdad de los ángulos bronquiales en los niños (13).

En cuanto a los hallazgos radiológicos más usuales en las obstrucciones bronquiales varían según las series. Puede observarse hiperinsuflación obstructiva, colapso, infiltrado, cuerpo extraño radiopaco, bronquiectasias con neumonitis local recurrente, neumomediastino y neumotórax. En un tercio de los casos, la radiografía es normal (1).

Como se ha dicho anteriormente, la mayoría de los cuerpos extraños aspirados son de origen vegetal y no se aprecian en la Rx. La realización de TAC no se recomienda de rutina, aunque puede ser útil en la identificación de los cuerpos extraños que no se visualizan mediante broncoscopia o la Rx tórax convencional (14).

\section{Bibliografía}

1. Mu L. Radiological diagnosis of aspirated foreign bodies in children: rewiew of 343 cases. J Laryngol Otol 1990; 104: 778-82.

2. Ortíz de Saracho J, García Leaniz J, Pérez Rodríguez E. Aspiración de un cuerpo extraño: Una etiología no tan infrecuente. Arch Bronconeumo 1994; 30: 323.

3. Blanco Aparicio M, Verea H, Martínez Lago V. Arch Bronconeumol 1994; 31: 93.

4. Hernández Martínez J, Ruiz López FJ, Martínez Garcerán JJ, Abellán Martínez MC, Méndez Martínez P, Sánchez Gascón F. Lesiones en el árbol traqueobronquial tras la aspiración de una tableta de sulfato ferroso. Arch Bronconeumol 1997; 33: 50-1.

5. Domingo Morera JA, Torres Nuez J, Sánchez Santos P, Mateo Lázaro ML, Bello Dronda S, Martínez Sanz G. Cuerpo extraño intrabronquial en adultos: Utilidad de la tomografía computarizada. Arch Bronconeumol 1997; 33: 201-3

6. Hilman BC, Kurzweg FT, McCook WW Jr et al: Foreign body aspiration of grass inflorescences as a cause of hemoptysis. Chest 1980; 78: 306-9.

7. Pneumocutaneous fistula secondary to aspiration of grass (letter) J. Pediatr 1973; 82: 737

8. Oberbauer B; Nicolai T; von Mutius. Bronchial asthma in chilhood.
Es indudable el interés diagnostico y terapéutico de la broncoscopia en estos casos. Sin embargo, el rendimiento de la broncoscopia es bajo si el cuerpo extraño se ha alojado en un bronquio de pequeño diámetro (3).

Al diagnóstico se llega a través de los antecedentes de la aspiración, la clínica, los hallazgos radiológicos y la broncoscopia. Si a pesar de todo este estudio no tuviéramos el diagnóstico, debería considerarse la realización de una biopsia a cielo abierto.

El microorganismo aislado en el aspirado bronquial Eike nella Corrodens, es una bacteria Gram negativo, anaerobio facultativo, que se encuentra en la flora normal orofaringea, genitourinaria y del aparato gastrointestinal. Las infecciones pulmonares son infrecuentes pero probablemente lo sean más de lo que se reconoce, porque no crece fácilmente en los medios de cultivo habituales (15). Respecto a la patología respiratoria ocasionada por este germen, se han descrito abscesos pulmonares, neumonías y cavitaciones. Estas infecciones son más frecuentes en situación de inmunodepresión. En la revisión bibliográfica realizada con Medline no hemos encontrado ninguna neumonitis por Eikenella Corrodens asociada a broncoaspiración de cuerpos extraños.

Es de destacar la utilidad de la broncoscopia y el lavado broncoalveolar como método de diagnostico y terapéutico. El LBA terapéutico se ha usado en la proteinosis alveolar, neumoconiosis, microlitiasis alveolar, mucoviscidosis, e inhalación de partículas radiactivas (16). Pensamos que en este caso pudo ayudar a la evacuación del cuerpo extraño. La eliminación espontánea ocurre en menos del $1 \%$ de los casos.

Lo inusual de este caso clínico es el largo periodo asintomático tras la aspiración de un cuerpo extraño de origen vegetal, así como la producción de neumonitis local por Eikenella Corro dens.

Part 1: Clinical manifestations, diagnosis and ground rules of therapy Fortschr Med 1993; 20, 111: 457-60.

9. Miller GA, Giarturco C, Neuky HG: The asymptomatic period in retained foreign bodies of the bronchus. AMA J Dis Child 1958; 95: 282-3.

10. Santiago S, Tobias J, Williams AJ: A reeppraisal of the causes of hemoptysis. Arch Inter Med 1991; 151: 2449-51.

11. Limpier AH. Tracheobronchial foreign bodies in adult. Ann Intern Med 1990; 112: 604-9.

12. Capitanio MA, Kirkpatric JA. The lateral decubitus film: An aid in determining air trapping in children. Radiology 1972; 103: 460-2.

13. Cleveland RH. Symmetry of bronchial angles in children. Radiology 1979; 133: 89

14. Berger PE, Kuhn JP, Kuhns LR: Computed tomografy an the occult tra cheobronchial foreign bodies. Radiology 1980; 134: 133-5.

15. Kentos A, De Vuyst P, Struelens MJ, Jacobs F: Lung Abscess due to Eikenelle Corrodens. Three cases and Review. Eur J Clin. Microbiol Infect Dis 1995; 14: 146-8.

16. Castella Riera J; Puzo Ardanuy C. Libro del Año. Neumología 1992 , Ediciones Saned, Madrid, 1992; 115-38. 